\title{
Improved the Response Throughput of Load Balancing of Scientific Cloud using Particle of Swarm Optimization
}

\author{
Rahul Bodkhe \\ M.Tech Scholar \\ Department of Information Technology \\ Samrat Ashoka Technological Institute \\ Vidisha(M.P),INDIA
}

\author{
Deepak Sain \\ Assistant Professor \\ Department of Information Technology \\ Samrat Ashoka Technological Institute \\ Vidisha(M.P) ,INDIA
}

\begin{abstract}
The efficiency and utility of cloud computing based on scheduling and balancing of load over cloud computing. The load balancing is important factor regarding the performance of cloud computing. Now a day's various heuristic function are used for the balancing and scheduling of load in cloud computing. Some heuristic function faced a problem of size of data and discontinuity of sequence of data. In this paper used particle of swarm optimization technique for the balancing of job in cloud environment. The nature of dynamicity of particle of swarm optimization supports the concept of dynamic load balancing technique. The modified load balancing algorithm simulate cloudsim simulator and used two other algorithm such as round robin and genetic algorithm. For the evaluation of performance cerate multiple size of job load matrix. Our experimental result shows that better performance instead of round robin and genetic algorithm.
\end{abstract}

\section{Keywords}

Cloud Computing, Load balancing, swarm intelligence, PSO.

\section{INTRODUCTION}

The scientific cloud computing processes are used in different discipline of engineering and technology for solving the problem related to earth science, bioinformatics and many more. The size of job for the processing of computer is very large and complex. The complex job and large size required the balancing of job for cloud computing. The load balancing technique divided the job on different virtual machine for the processing of data. The allocation and scheduling of job over virtual machine play an important role in efficiency of cloud computing [6]. For the scheduling of Job and allocation of virtual machine previously used conventional technique such as round robin and FCFS technique. This conventional technique is not sufficient for the processing of large and complex job. For the better improvement of load balancing process used dynamic load balancing technique. The dynamic load balancing technique used swarm intelligence [13]. The swarm intelligence technique various optimization algorithm such as genetic algorithm, ant colony optimization, particle of swarm optimization. The particle of swarm optimization algorithm is basically based on dynamic based population technique. The dynamic population technique proceeds the job on the basis of local and global optimal. The allocation and management process of particle of swarm optimization is better than other heuristic function. The modified particle of swarm optimization algorithm maintains the parallel execution of task during the processing of job loading.
The particle of swarm optimization creates here two search space one for load for allocation VM and other is searching an allocated job of particular virtual machine. The allocated virtual machine load transfer to another machine that status is free for the execution of job [8]. The rest of paper describe as section II discuss load balancing. in section III discuss related work. In section IV we discuss the modified algorithm. In section $\mathrm{V}$ discuss the experimental evaluation of particle of swarm optimization and finally discuss conclusion and future work in section VI.

\section{LOAD BALANCING}

Load balancing policy and technique provide a great facility in cloud computing for the processing of job and balancing of load. The provisioning of resource and user request in multiple constraints of job creates the load of the system. The proper scheduling of job increases the efficiency of cloud computing. The cloud computing infrastructure delivers various types of services over internet. The demand of services and user request creates some issue in terms of balance factor.

- The process of cloud computing offer on demand services.

- The utilization of resource depends on the condition of balance factor of load.

- The process of load is low lots of resource is minimum.

- Reduces the cost factor in terms of balancing process.

For maintaining the load balancing process cloud computing uses two different technique such as static and dynamic. The static load balancing technique follows the CPU scheduling algorithm. The CPU scheduling algorithm balance the load in limited resource, but the environment of cloud computing offer various types of resource as a services such as IAAS,PAAS and SAAS. For the balancing purpose of load over cloud network the traditional and conventional process of balancing is not sufficient. For the improvement of load balancing technique used dynamic scheduling technique. the dynamic load balancing technique used swarm intelligence. The swarm intelligence technique offers various types of algorithm such as particle of swarm optimization, ant colony optimization and many more swarm derive function.

\section{RELATED WORK}

In this section we define the rich literature review in the field of load balancing techniques in cloud computing environment on the basis of various journal and research papers.

[1] In this paper Author discuss the about the cloud computing load mechanism and virtualization techniques for the improvement of performance over cloud computing. Authors 
also describe the process of job selection and scheduling of task. For the selection of task used swarm based optimization. The swarm based optimization technique optimized provides the load dynamic concept.

[2] In this paper authors define the dynamic load balancing in clod computing environment. Authors describe the scheduling of load as CPU. Basically CPU based operation used limited number of resource for the management of resource, these concept applied over the cloud network the performance are decrees in terms of throughput. Here authors used the hash code technique for the allocation of process.

[3] In this paper author proposed a cloud job scheduler based on ant colony optimization, they scheduler accomplished the process of selection of job in different unit of segment. The Job scheduler work in collection of distributed job in consequence of iteration of balancing factor.

[4]In this paper author proposed a ant based job scheduler for the management of job in cloud environment. The ant based job scheduler based on the principle of ant colony optimization technique. the ant colony optimization technique inspired by real ants behavior. The proposed scheduler better than traditional and conventional cloud job scheduler. The maximization of job the ant scheduler behaves like normal cloud scheduler.

[5] In this paper author describe the techniques such as swarm intelligence (SI), ant colony optimization (ACO) and particle swarm optimization (PSO) for the cloud based scheduler mechanism, to allocate VM to physical Cloud resources. Simulated experiments performed by using CloudSim and real PSE job data suggest that their schedulers, through a weighted metric, perform competitively with respect to the number of serviced users and achieve an effective assignment of VMs compared to a scheduler based on Genetic Algorithms.

[6] In this paper author define ACO techniques for the scheduling problem in hybrid clouds presenting the main Heuristics such as cost, makesapn, number of cores (multicore), and available bandwidth to be considered when scheduling workflows. ACO is one of the best optimization techniques in scheduling workflows using heuristics based mechanism. Schedulers for cloud computing determine on which processing resource jobs of a workflow should SS be allocated. In hybrid clouds, jobs can be allocated on either a private cloud or a public cloud on a pay per uses basis.

[7] In This paper they proposed the improve particle swarm optimization algorithm in resources scheduling strategy of the cloud computing. Through experiments, the results show that this method can reduce the task average running time, and raises the rate availability of resources. Here the assign of Job scheduling problem is a core and challenging issue in cloud computing environment.

[9] In this paper author define here to the best of their knowledge; existing efforts do not address in general online

clouds where multiple users are connected to execute their scientific experiments. On the other hand, no effort aimed at balancing the number of serviced users in a Cloud and the total number of created VMs by the scheduler exists. In fact, the greater the number of serviced users, the better the throughput, and the more created VMs, the higher the achieved parallelism. More parallelism means executing a greater number of jobs, and hence a more agile human processing of PSE job results.
[11] in this paper authors discuss the application and diversity of swarm intelligence in cloud and grid computing. Basically authors used the process of load balancing and job scheduling for allocation and distributed of resource. For the allocation and distribution of resource used different swarm based algorithm such as fish fork and other kits behaviors

\section{MODIFIED ALGORITHM}

The particle of swarm optimization selects the input of virtual load in terms of $D^{R}$ the $\mathrm{D}$ show the value of domain load and $\mathrm{R}$ shows that Job component of $\mathrm{R}$ load. The R Job content $\{\mathrm{r} 1, \mathrm{r} 2, \mathrm{r} 3, \mathrm{r} 4, \ldots \ldots . \mathrm{rn}\}$ describe the artificial particle as population. The unique job relation of input load set the velocity of particle. If the job attribute value is change then next iteration moves the update of velocity. These terms describe as, the particle's job value, Rid and its near value of particle; Rgd is a velocity value of optimization job space. The random values for job are fet 1 and fet 2 are used for the local and global value selection of particle, that is, to make the optimal solution. The values of $\mathrm{c} 1$ and $\mathrm{c} 2$ manage the value of velocity of Rid and Rgd in deciding the particle's next movement velocity. At that each iteration changes the velocity of swarm and creates new job subset for selection of job. The derivation of equation in (c) and (d) [9]

$$
\begin{gathered}
v_{i d=W \times V_{i d}+C 1 \times f e t} 1 \times\left(R_{i d-x_{i d}}\right)+C_{2} \times \text { rand } 2 \times\left(R_{g d}-X_{i d}^{c}\right) \ldots \ldots .(c) \\
X_{i d=X_{i d}+V_{i d} \ldots \ldots \ldots \ldots \ldots \ldots \ldots \ldots . .(d)}
\end{gathered}
$$

where $w$ denotes the value of job matrix; Rid is the position of particle value, Rgd is the position of global value best fitness value, $\mathrm{c} 1$ and $\mathrm{c} 2$ are constants and are known as acceleration coefficients; $d$ denotes the dimension of the problem space; fet 1 , fet 2 are random values in the range of $(0,1)$. The finally optimal job are executed to data centre. Here first define the fitness constraints function for the allocation of job corresponding to virtual machine. here define the Mi is the set of virtual machine and $\mathrm{Ri}$ is the set of job and $\mathrm{Lk}$ is total load over the cloud. The fitness constraints function define as

$$
\begin{aligned}
& L_{k}\left(M_{i, R_{j}}\right) \\
& =\frac{\left[\tau\left(M_{i}, R_{j}\right)\right] \alpha\left[\rho\left(M_{i}, R_{i}\right)\right] \beta}{\sum_{h \in l k}(M i, R j)^{[\tau(M i, h)] \alpha[\rho(R i, h)] \beta}}, \operatorname{Ri} \in \operatorname{Lk}(M i, R i) \ldots
\end{aligned}
$$

Here $\tau(M i, R i)$ is the partial load and available virtual machine.

Here discuss the steps

1. Procedure LOADPSO ()

2. Begin

3. Initialize resource particle $(\mathrm{R} 1, \mathrm{R} 2$ Rn)

4. Vmindex $=0$

5. Do

6. $\mathrm{Map}(\mathrm{Mi}, \mathrm{Ri})$

7. While(joblist.size ()$>0)$

8. Job=joblist.getnextjob()

9. End

10. if $($ job $=$ resource $)$

11. \{allocation process is done

12. 

13. Else
14. Search new virtual machine
15. If (velocity=new location)
16. \{update position of job
17. \}
18. Else
19. Process is terminated.

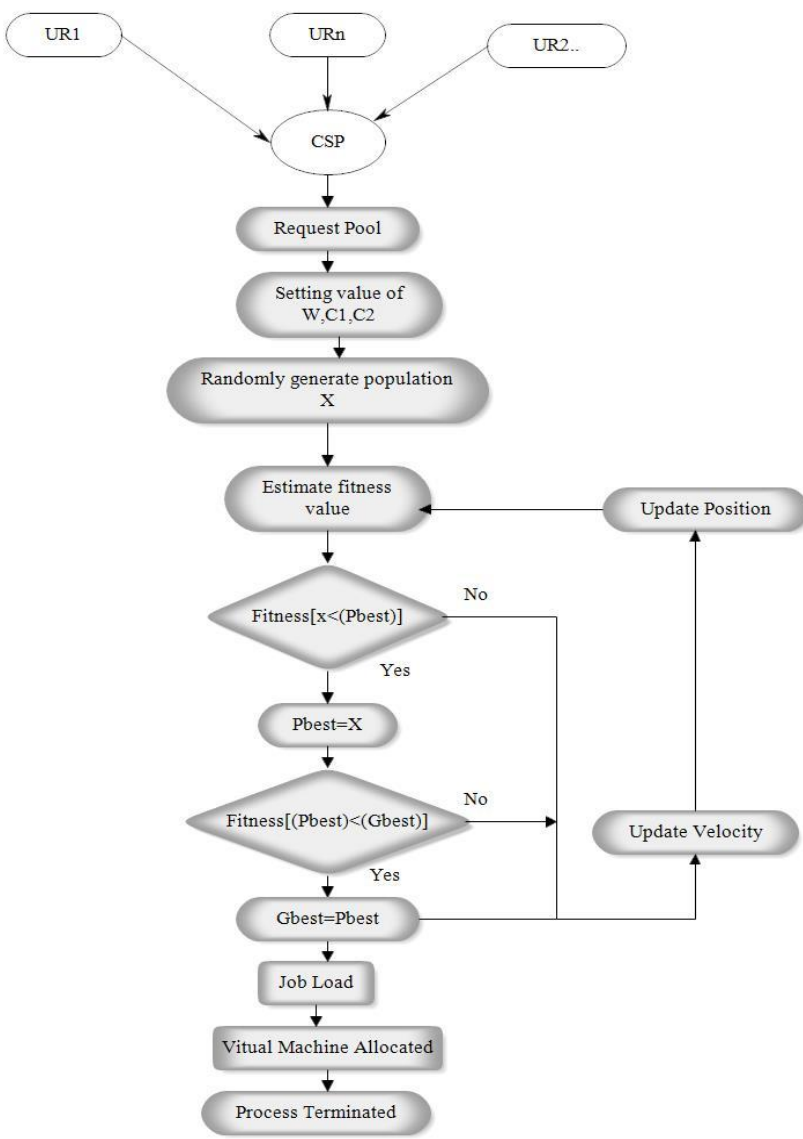

Figure 1 process block diagram of load balancing process based on PSO

\section{EXPERIMENTAL RESULT ANALYSIS}

In this section we define the experimental results analysis with the various techniques such as RR, GA and PSO.

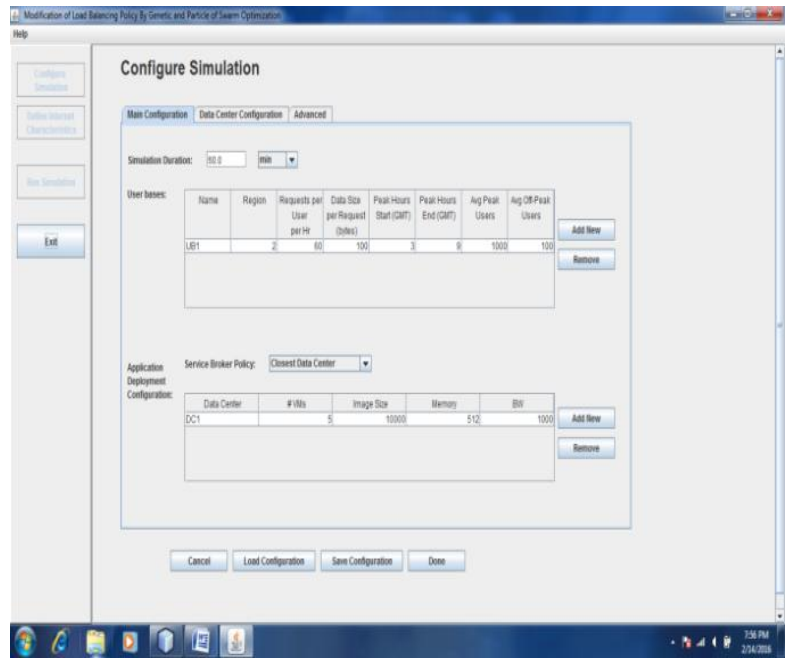

Figure 1: Shows that the main Simulation window for cloud computing analyst.

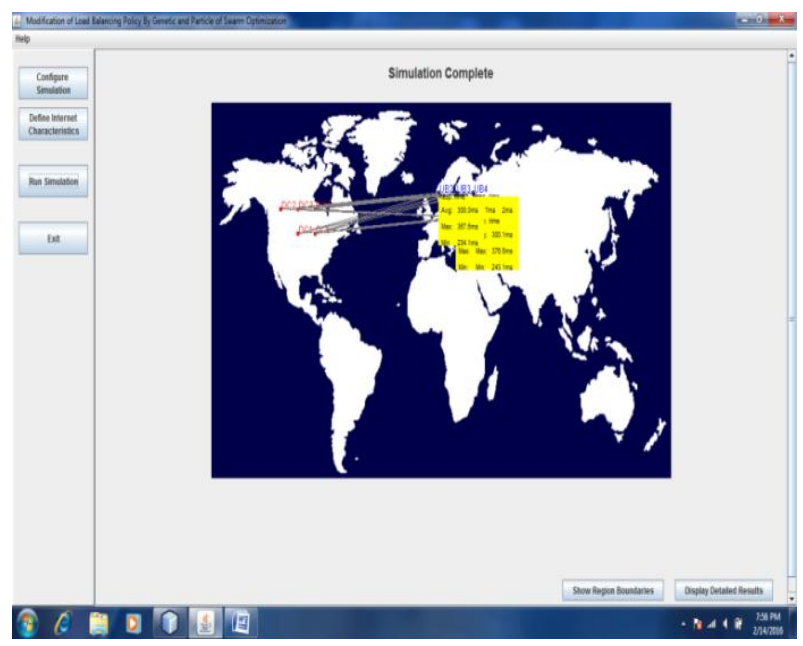

Figure 2: Shows that the result window for cloud computing

Data centre connection using Round Robin method with input value is 5 .

Table 1: Comparative performance evaluation using Round Robin, Genetic Algorithm and particle of Swarm Optimization methods with the input value is 5 .

\begin{tabular}{|l|l|l|l|l|}
\hline $\begin{array}{l}\text { Number } \\
\text { of Input }\end{array}$ & $\begin{array}{l}\text { Method } \\
\text { Name }\end{array}$ & $\begin{array}{l}\text { Average } \\
\text { Time }\end{array}$ & $\begin{array}{l}\text { Minimum } \\
\text { Time }\end{array}$ & $\begin{array}{l}\text { Maximum } \\
\text { Time }\end{array}$ \\
\hline \multirow{5}{*}{5} & $\begin{array}{l}\text { Round } \\
\text { Robin }\end{array}$ & 0.31 & 0.02 & 0.62 \\
\cline { 2 - 5 } & $\begin{array}{l}\text { Genetic } \\
\text { Algorith } \\
\text { m }\end{array}$ & 0.22 & 0.01 & 0.44 \\
\cline { 2 - 5 } & PSO & 0.16 & 0.01 & 0.31 \\
\hline
\end{tabular}


Table 2: Comparative performance evaluation using Round Robin, Genetic Algorithm and particle of Swarm Optimization methods with the input value is 10 .

\begin{tabular}{|l|l|l|l|l|}
\hline $\begin{array}{l}\text { Number } \\
\text { of Input }\end{array}$ & $\begin{array}{l}\text { Method } \\
\text { Name }\end{array}$ & $\begin{array}{l}\text { Average } \\
\text { Time }\end{array}$ & $\begin{array}{l}\text { Minimum } \\
\text { Time }\end{array}$ & $\begin{array}{l}\text { Maximum } \\
\text { Time }\end{array}$ \\
\hline \multirow{2}{*}{10} & $\begin{array}{l}\text { Round } \\
\text { Robin }\end{array}$ & 0.36 & 0.02 & 0.69 \\
\cline { 2 - 5 } & $\begin{array}{l}\text { Genetic } \\
\text { Algorithm }\end{array}$ & 0.24 & 0.01 & 0.43 \\
\cline { 2 - 5 } & PSO & 0.18 & 0.01 & 0.34 \\
\hline
\end{tabular}

\section{Comparative result graph Cloud computing} load balancing with the RR, GA and PSO methods for the input value is 5

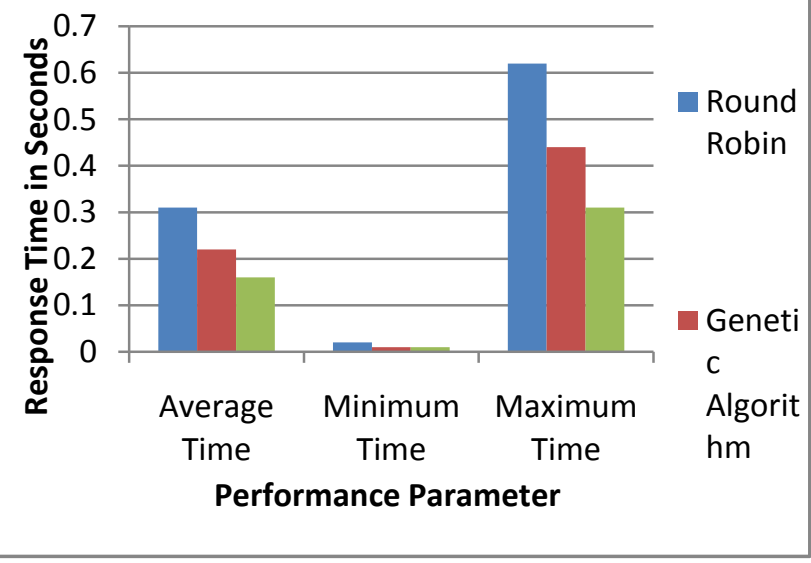

Figure 3: Shows that the comparative performance evaluation for each method with the different numbers of input values here the input value is 5 .

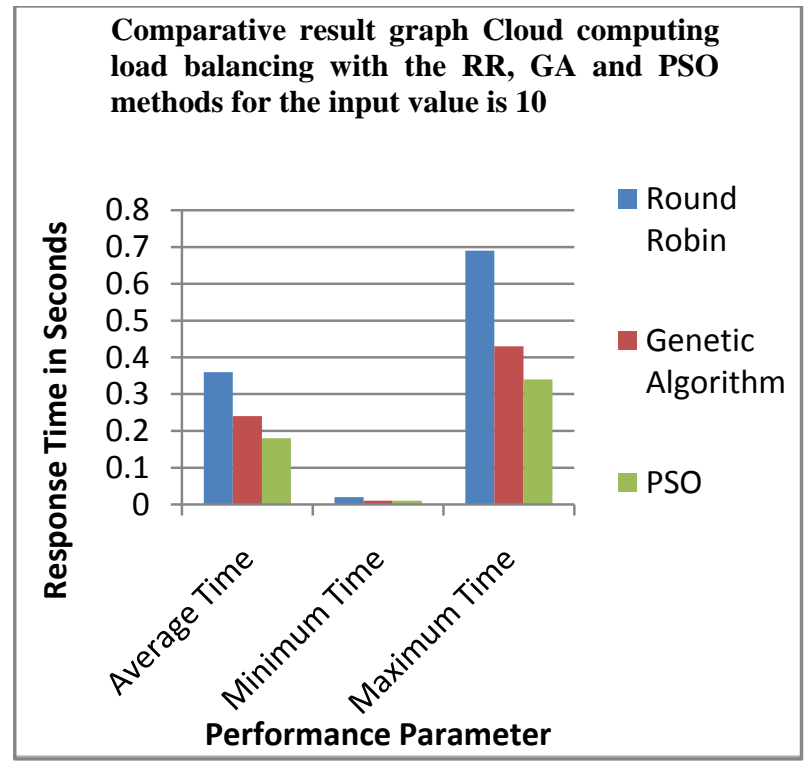

Figure 4: Shows that the comparative performance evaluation for the cloud computing load for each different number of input value here the input value is $\mathbf{1 0}$.

\section{CONCLUSION AND FUTURE SCOPE}

In this paper used particle of swarm optimization algorithm for the selection of job and task for the resource allocation in public cloud computing. The PSO function gives the better performance instead of other swarm based algorithm such as glowworm swarm intelligence algorithm. Basically PSO algorithm facilities the all task in two sections one is job allocation process and other is job selection process. The job selection process is defined by the predefined constraints function. For the evaluation of performance used cloud simulator software such is called cloud analyst. The cloud analysis software is bag of composition of cloud environment and load balancing policy. In scenario of policy design two services one is genetic algorithm policy and other is PSO based policy. The PSO based policy reduces the load effect approx $10-12 \%$ in compression of genetic algorithm. the modified load balancing policy is very efficient for scientific cloud computing.

\section{REFERENCES}

[1] Elina Pacini, Cristian Mateos, Carlos García Garino "Balancing Throughput and Response Time in Online Scientific Clouds via Ant Colony Optimization" Elsevier. 2014 PP1-37.

[2] Ratan Mishra, Anant Jaiswal "Ant colony Optimization: A Solution of Load balancing in Cloud" International Journal of Web \& Semantic Technology 2012 PP 33-50.

[3] Cristian Mateos , Elina Pacini, Carlos García Garino "An ACO-inspired Algorithm for MinimizingWeighted Flowtime in Cloud-based Parameter Sweep Experiments" Elsevier. 2013 PP. 38-50.

[4] Carlos GARCÍA GARINO, Cristian MATEOS , Elina PACINI "ACO-based dynamic job scheduling of parametric computational mechanics studies on Cloud Computing infrastructures" IARW 2012.

[5] Elina Pacini , Cristian Mateos , Carlos García Garino "SI-based Scheduling of Scientific Experimentson Clouds" 2013. IEEE

[6] J.Elayaraja, S.Dhanasekar "A Survey On Workflow Scheduling In Cloud Using Ant Colony Optimization" IJCSMC 2014 PP.39 - 44.

[7] Shaobin Zhan, Hongying Huo "Improved PSO-based Task Scheduling Algorithm in Cloud Computing" Journal of Information \& Computational Science 2012 PP 3821-3829.

[8] Lu Huang, Hai-shan Chen, Ting-ting Hu "Survey on Resource Allocation Policy and Job Scheduling Algorithms of Cloud Computing" Journal Of Software, 2013 PP 480-487.

[9] Elina Pacini - Cristian Mateos - Carlos García "Multiobjective Swarm Intelligence Schedulers for Online Scientific Clouds" Springer. 2014 PP 1-35.

[10] Soumya Banerjee, Indrajit Mukherjee, P.K. Mahanti "Cloud Computing Initiative using Modified Ant Colony Framewor" International Scholarly and Scientific Research \& Innovation 2009 PP 1962 -1965.

[11] R. Buyya, C. Yeo, S. Venugopal, J. Broberg, I. Brandic, "Cloud Computing And Emerging It Platforms: Vision, Hype, And Reality For Delivering Computing" The 5th Utility, Future Generation Computer Systems 2009 PP 599-616. 
[12] R. Calheiros, R. Ranjan, A. Beloglazov, C. De Rose, R. Buyya "Cloudsim: A Toolkit For Modeling And Simulation Of Cloud Computing Environments And Evaluation Of Resource Provisioning Algorithms", Software: Practice \& Experience 2011 PP 23-50.

[13] El-Rewini, H., Ali, H.H., Lewis, T. "Task Scheduling In Multiprocessing Systems”, IEEE, 1995, PP. 27-37.
[14] E. D. Lumer , B. Faieta, "Diversity And Adaptation In Populations Of Clustering Ants," Int. Conf. Simulation Adaptive Behavior, 1994, PP. 501-508.

[15] Peter S. Pacheco, "Parallel Programming With MPI", Morgan Kaufmann Publishers Edition 2008.

[16] Mequanintmoges, Thomas G.Robertazzi, "Wireless Sensor Networks: Scheduling For Measurement And Data Reporting”, August 31, 2005. 